American Journal of Engineering and Applied Sciences 4 (4): 598-606, 2011

ISSN 1941-7020

(C) 2014 S. Idarwazeh, This open access article is distributed under a Creative Commons Attribution

(CC-BY) 3.0 license

\title{
Inverse Discrete Fourier Transform-Discrete Fourier Transform Techniques for Generating and Receiving Spectrally Efficient Frequency Division Multiplexing Signals
}

\author{
Sahmed Idarwazeh \\ Department of Electronic and Electrical Engineering, \\ University College London, WC1E 7JE, UK
}

\begin{abstract}
Problem statement: Spectrally Efficient Frequency Division Multiplexing (SEFDM) system promises bandwidth savings by multiplexing overlapped non-orthogonal sub-carriers. However, the loss of orthogonality results in increasing the complexity of generation and detection of the signal. In this work, we propose simple framework for the generation of the SEFDM signal based on the Inverse Discrete Fourier Transform (IDFT). Approach: This study further proposes the use of the Discrete Fourier Transform (DFT) as the receiver front end, specifically for extracting the statistics of the signal needed for recovering the transmitted signal. The proposed transmitter designs employ similar building blocks as Orthogonal Frequency Division Multiplexing (OFDM) based systems, hence, would facilitate an easy migration and/or coexistence with OFDMon the transmitter side. Furthermore, the proposed framework may facilitate the IDFT design for any Frequency Division Multiplexed (FDM) signal with arbitrary sub-carriers spacing. Results and Conclusion: The equivalence of the IDFT generated signal and the modulators based signal is proved mathematically, nonetheless, numerical simulations were performed to verify that equivalence and to test for the performance of the digitally generated signal in AWGN channel. Numerical results confirmed the required spectrum compression and Bit Error Rate (BER) performance at a much reduced complexity.
\end{abstract}

Keywords:Required spectrum, bit error rate, numerical results, digitally generated, generated signal, spectrally efficient, division multiplexing, transmitted signal, proposed transmitter, similar building

\section{INTRODUCTION}

Demand from wireless communications has always necessitated a search for techniques to save the precious wireless spectrum. Orthogonal Frequency Division Multiplexing (OFDM) has been proposed as a multi carrier scheme that provides immunity against the frequency selective channel, therefore, enabling efficient utilization of the spectrum (Weinstein and Ebert, 1971; Raleigh and Cioffi, 1998; Hanzo, 2003) and (Hanzo and Keller, 2006). In OFDMthe subcarriers are placed at specific locations to satisfy the orthogonality rule. Recently, Spectrally Efficient Frequency Division Multiplexing (SEFDM) system is proposed as a system that promises better utilization of bandwidth by reducing the spacing of the sub-carriers. Higher spectral efficiency is achieved by relaxing the orthogonality rule, defined for OFDM, to allow the carriers to be placed at closer frequency separation. In fact, the concept of non-orthogonal multi carrier systems has appeared under different scenarios with a common goal to increase spectral efficiency yet with different architectures. Fast OFDM (Rodrigues and Darwazeh, 2002) and M-ary Amplitude Shift Keying (ASK) OFDM (Xiong, 2003) are two systems that independently suggested bandwidth savings of $50 \%$ of the OFDM bandwidth for one dimensional modulation schemes. In addition, a system that proposes a similar concept as SEFDM termed High Compaction Multi Carrier (HC-MC) system appeared in (Hamamura and Tachikawa, 2004). HC-MC achieves spectral efficiency by reducing transmission time of an equivalent OFDM system. Moreover, (Rusek and Anderson, 2005; 2009) proposed the so called Multistream Faster than Nyquist Signaling (FTN) promising enhanced spectral utilization backed up by the study in Mazo (1975), where Mazo established that it is possible to increase the signaling rate by $20 \%$ without experiencing any performance degradation. The main differences between all these systems is the architecture of the system proposing spectral efficiency.

Work in the literature has confirmed that despite the non-orthogonal structure of the sub-carriers, efficient detection of the SEFDM signal is achievable. 
However, loosing orthogonality brings along many design and implementation challenges. In particular, the generation of the signal requires a bank of independent modulators which becomes particularly complex with the increase in the number of carriers whilst the detection of the signal needs to account for the intercarrier interference due to the loss of orthogonality. Maximum Likelihood (ML) is shown to provide matching performance to OFDM in AWGN channel (Rodrigues and Darwazeh, 2003). Nevertheless, ML detection is particularly complex, with a computational complexity that grows exponentially with the size of the system. In addition, due to the intercarrier interference in the system linear detection techniques such as Zero Forcing (ZF) and MinimumMean Square Error (MMSE), failed to achieve bit error rates that can compete with todays wireless applications requirements (Kanaras et al., 2008a; 2008c). However, the application of Sphere Decoding algorithm has confirmed that SEFDM can achieve optimum BER performance with a much reduced complexity (Kanaras et al., 2008b; 2009). Overall, research in SEFDM system has confirmed that detection of SEFDM signals corrupted with additive white Gaussian noise is viable. Systems providing bandwidth savings of up to $25 \%$ are proposed without any error penalty compared to OFDM. In addition, (Chorti et al., 2010) has demonstrated that the SEFDM system is capable 2 of delivering attractive performance in fading channels.

Aside from the complex detection, SEFDM system is limited by its transmitter structure. SEFDM transmitter consists of a bank of modulators running at the sub-carriers frequencies. For large system size it becomes exceptionally complex to have this bank of modulators. In addition, the system will be more susceptible to frequency offsets and timing errors, as the number of oscillators increases. Kanaras et al. (2009) the use of Fractional Fourier Transform (FrFT) for SEFDM signal generations was proposed. The FrFT can be realized with efficient algorithms based on the Bluestein algorithm for the Fast Fourier Transform evaluation. However, this algorithm requires at least three modules of the Inverse Discrete Fourier Transform (IDFT) of length of double the number of carriers and pre-processing in the form of rotations (Bailey and Swarztrauber, 1991). In contrast, OFDM signal is efficiently generated with a single IDFT (Salz and Weinstein, 1969; Weinstein and Ebert, 1971). In this study we propose simple IDFT implementations to generate signals with fractional frequency spacing. The framework proposed here requires minor changes in the input symbols stream to facilitate the IDFT design of any signal with an arbitrary subcarriers spacing such as SEFDM and M-ary Amplitude Shift Keying (ASK)
OFDM system proposed in (Xiong, 2003). The proposed transmitters facilitate the digital generation of the SEFDM signal which can be received using receivers structures as in (Rodrigues and Darwazeh, 2003; Kanaras et al., 2008b; 2009). The performance of the digitally generated signal is verified by simulations. Furthermore, the framework is extended to provide simplified SEFDM receiver front end based on the Discrete Fourier Transform (DFT).

The rest of this study is organized as follows: Spectrally Efficient FDM system is briefly outlined. The IDFT implementations of SEFDM transmitter is presented. Section extends the study to propose simple DFT based correlators for the SEFDM receiver. Section evaluates the proposed transmitters in terms of computational complexity and applicational advantage. The study is concluded in section.

Spectrally efficient FDM signals: SEFDM signal is constructed by modulating blocks of the input data stream on parallel carriers (Rodrigues and Darwazeh, 2003), as shown in Fig. 1. The input data stream is represented as complex symbols denoted by $\mathrm{s}=\mathrm{s}_{\mathfrak{g l}}+\mathrm{js} \mathfrak{I}_{\mathrm{y}}$ to denote QAM symbols. The Serial to Parallel (S/P) converter groups the input symbols in blocks and modulates each block over the nonorthogonal carriers. At the receiver, the incoming signal is projected over orthonormal bases and detected using detection algorithms such as ML or SD.

The carriers in SEFDM systems are spaced by a fraction of the inverse of the symbol duration denoted as $\alpha$ where $\alpha=\Delta \mathrm{fT}, \Delta \mathrm{f}$ is the frequency distance between the sub-carriers and $\mathrm{T}$ is the symbols duration. Thus the SEFDM carriers violate the orthogonality condition of OFDM systems where the spacing is equal to the inverse of the symbol duration. The spectral efficiency improvement of the SEFDM signal over the OFDM approaches $1 / \alpha$ with the increase in the number of carriers. Figure 2 depicts the subcarriers of the SEFDM system and OFDM System to illustrate how the spectrum is compressed in SEFDM system.

Equation 1 gives the time domain SEFDM signal as $x(t)$ in complex baseband representation, where $N_{c}$ is number of sub-carriers and $s_{1, n}$ is the symbol modulated on the $\mathrm{n}_{\text {th }}$ sub-carrier in the $1^{\text {th }}$ SEFDM frame:

$$
\chi(t)=\frac{1}{\sqrt{T}} \sum_{\mathrm{l}=-\infty}^{\infty} \sum_{\mathrm{n}=0}^{N_{c}-1} \mathrm{~s}_{1, \mathrm{n}} \exp \left(\mathrm{j}^{2} \pi \mathrm{n} \alpha(\mathrm{t}-\mathrm{IT}) / \mathrm{T}\right)
$$

Consider the first SEFDM frame from (1), sampled at (T/N) intervals where $N \geq N_{c}$, the frame is expressed in (2)for $\mathrm{k}=0,1, \cdots, \mathrm{N}-1$, where $\mathrm{s}_{\mathrm{n}}$ denotes $\mathrm{n}^{\text {th }}$ input symbol in the frame under consideration. $X[\mathrm{k}]$ is $\mathrm{k}^{\text {th }}$ timesample of the first frame of $x(t)$ in (1). The factor $1 / \sqrt{\mathrm{N}}$ in (2) is for normalization purpose: 
Am. J. Engg. \& Applied Sci., 4 (4): 598-606, 2011

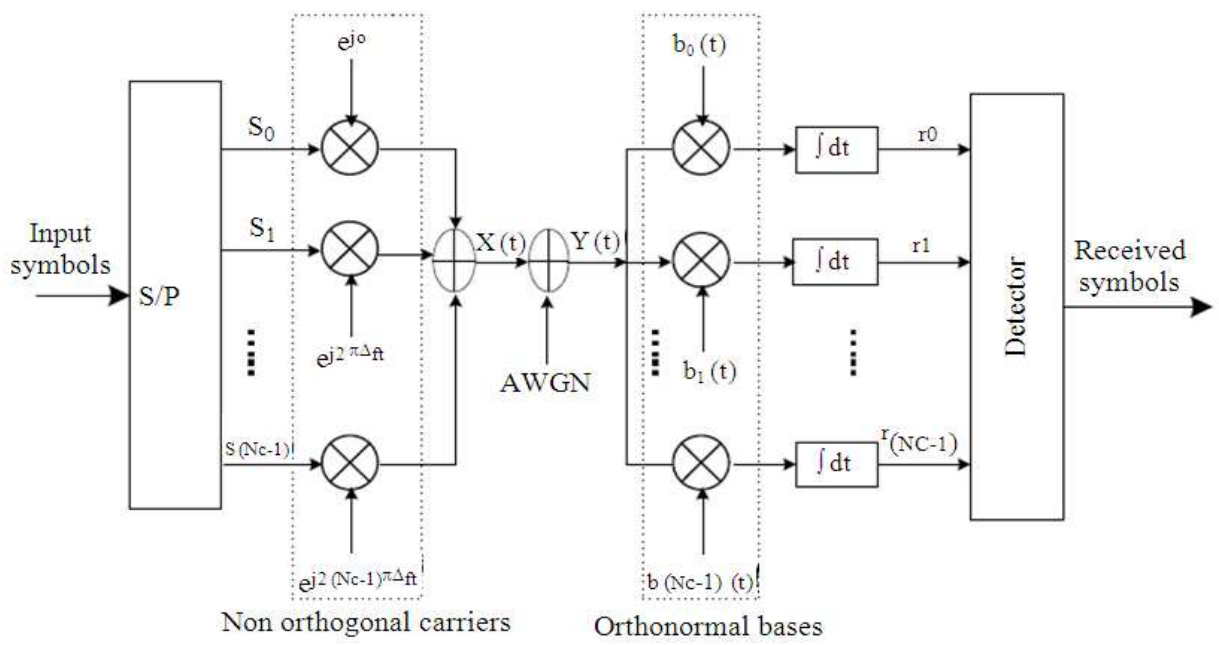

Fig. 1: The SEFDM system model

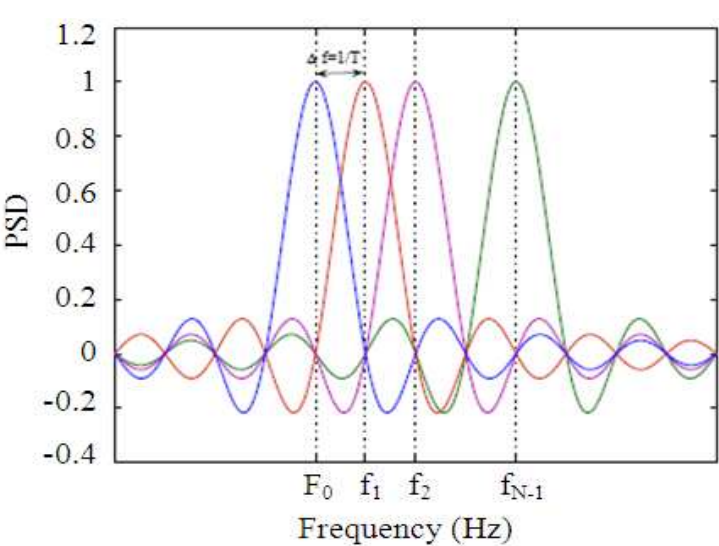

(a)

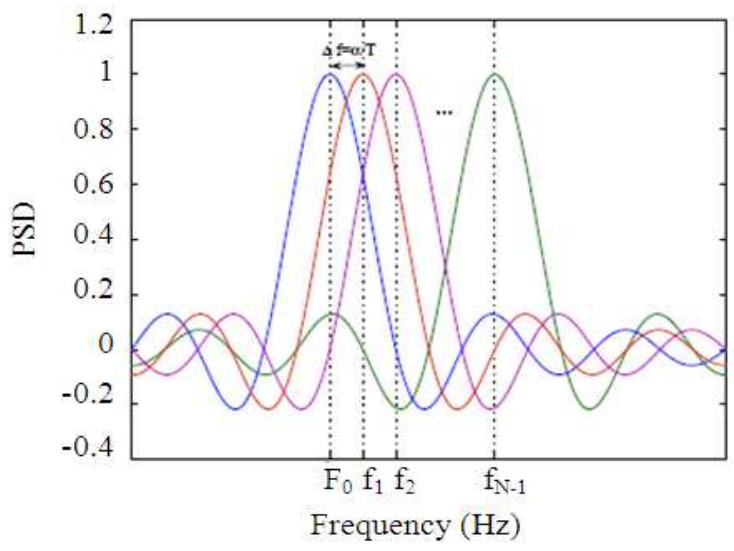

(b)

Fig. 2: OFDM carriers (left) and SEFDM carriers right) SEFDM carriers fill narrower bandwidth while delivering the same data rate to the end point
$\mathrm{X}[\mathrm{k}]=\frac{1}{\sqrt{\mathrm{N}}} \sum_{\mathrm{n}=0}^{\mathrm{N}-1} \mathrm{~s}_{\mathrm{n}} \exp \left(\frac{\mathrm{j} 2 \pi \mathrm{nk} \alpha}{\mathrm{N}}\right)$

The system in (2) can be expressed in matrix form as Eq. 3:

$\mathrm{X}=\Phi S$

where, $\mathrm{X}=\left[\mathrm{x}_{0}, \cdots, \mathrm{X}_{\mathrm{N}-1}\right]^{\prime} 0$ is a vector of transmitted samples of $\mathrm{X}(\mathrm{t})$ in $(1)$ and $\mathrm{S}=\left[\mathrm{s}_{0}, \cdots, \mathrm{s}_{\mathrm{Nc}-1}\right] 0$ is a vector of input symbols , [.]' denotes a vector or matrix transpose operation. The sampled SEFDM carriers are given by he matrix $\Phi$ where $\Phi$ is an $\mathrm{N}_{\mathrm{c}} \times \mathrm{N}$ matrix whose elements are given by $\Phi_{\mathrm{n}, \mathrm{k}}(1 / \sqrt{\mathrm{N}}) \exp$ $(\mathrm{j} 2 \pi \alpha n \mathrm{n} / \mathrm{N})$ for $0 \leq \mathrm{n} \leq \mathrm{N}_{\mathrm{c}}-1,0 \leq \mathrm{k} \leq \mathrm{N}-1$.

Figure 1 depicts the SEFDM receiver as proposed in (Rodrigues and Darwazeh, 2003). The receiver employs a bank of correlators that collects sufficient statistics from the received signal. This is achieved by projecting the signal onto orthonormal bases (Proakis and Salehi, 2008).

Let the received signal be denoted by y $(\mathrm{t})$, where Eq. 4:

$y(t)=x(t)+w(t)$

w (t) an additive white Gaussian noise term. The $i^{\text {th }}$ collected statistics $r_{\mathrm{i}}$ is obtained as Eq. 5:

$r_{i}=\int_{0}^{T} y(t) b_{i}(t)$

The bases $b_{i}$ (t) are orthonormal in order to preserve the white nature of the noise in the system. However, projection onto a set of basis different from 
the one used for signal generation means that the intercarrier interference is not eliminated. The complete set of received statistics is then described as Eq. 6:

$$
\mathrm{R}=\mathrm{LS}+\mathrm{W}
$$

where, the correlation between the orthonormal bases and the original carriers is denoted by the $N_{c} \times N_{c}$ matrix $\mathrm{L}$ and $\mathrm{R}, \mathrm{S}$ and $\mathrm{W}$ are $\mathrm{N}_{\mathrm{c}} \times 1$ statistics vector, transmitted symbols vector and noise samples vector respectively. The $\mathrm{L}$ matrix is found to be upper triangular confirming the existence of intercarrier interference (Kanaras et al., 2008a). The properties of matrix L determines the quality of the obtained statistics and also determines the detection mechanism. The sufficient statistics $\mathrm{R}$ are fed to a detector that employs complex detection algorithms to extract the transmitted signal with the aim of minimizing the Bit Error Rate (BER) as outlined.

As the focus of this study is on simple ways to generate the SEFDM signal the rest of this correspondence will be dedicated for the design and performance verification of simple digital SEFDM transmitters based on the standard IDFT operation. Furthermore, the proposed framework for generating SEFDM signals is extended to provide $\mathrm{s}$ implecorrelators in the first stage of the receiver.

The IDFT Based SEFDM Transmitter: SEFDM signal is generated using a bank of modulators running at the sub-carriers frequencies. For large system size the complexity of the analogue system increases dramatically. In addition, there will be a serious constraint on the frequency precision of the oscillators in the system. In contrast, OFDM signal is efficiently implemented using IDFT. For an $\mathrm{N}$ carrier OFDM system, the input symbols are fed into a $\rho \mathrm{N}$ point IDFT, where $\rho$ is the number of samples per carrier, then the outputs of the IDFT are fed into a digital to analogue converter to generate the continuous time domain signal (Weinstein and Ebert, 197; Macedo and Sousa, 1997). We will show how the IDFT can be used to generate non-orthogonal signals such as the SEFDM signal, where the IDFT of a sequence $\mathrm{Y}=\left[\mathrm{y}_{0}, \mathrm{y}_{1}, \cdots, \mathrm{y}_{\mathrm{N}-1}\right]$ ' denoted with the vector $\mathrm{F}^{-1}\{\mathrm{Y}\}$ is given by Eq. 7 (Oppenheim et al., 1999):

$$
F_{k}^{-1}\{Y\}=\frac{1}{\sqrt{N}} \sum_{n=0}^{N-1} y_{n} \exp \left(\frac{j 2 \pi n k}{N}\right)
$$

Consider the SEFDM signal as in (3) and extend the input signal vector to length $\mathrm{M}$ by appending $\mathrm{M}-\mathrm{N}$ zeros such as Eq. 8:

$$
S^{\prime}=\left\{\begin{array}{cc}
S_{i} & 0 \leq i<N \\
0 & N \leq i<M
\end{array}\right.
$$

The definition of $\mathrm{M}$ is crucial to the IDFT representation of the SEFDM signal. Assuming that N/ $\alpha$ is an integer, the SEFDM signal can be expressed as:

$$
X^{\prime}=(1 / \alpha) Q^{\prime}
$$

such that $X\left[k^{\prime}\right]=X^{\prime}\left[k^{\prime}\right]$ for $1<k^{\prime}<N, Q$ is an $M \times M$ IDFTmatrixwith entries $q_{n, k}=\frac{1}{\sqrt{M}} \exp \left(\frac{j 2 \tau n k}{M}\right), 0 \leq n, k$ $\leq \mathrm{M}-1, \mathrm{M}>\mathrm{N}$ and $\mathrm{M}=\mathrm{N} / \alpha$ to establish the equivalence between (3) and (9).

Equation 9 expresses the SEFDM signal as the first $\mathrm{N}$ outputs of the IDFT of a modified input symbols vector. These $\mathrm{N}$ outputs represent time samples of the SEFDM signal; therefore, can be fed into a digital to analogue converter to generate the signal in time. Figure 3 depicts atransmitters for the case $N / \alpha$ is an intege $r$. The change in the size of the input symbols vector ensures that the frequency samples of the SEFDM system coincides only with the frequency samples of the IDFT that are within the bandwidth of the SEFDM signal. The reduction in bandwidth offered by SEFDM system is achieved by suppressing the unwanted frequency tones by setting the corresponding inputs to zero.

If more than $\mathrm{N}$ outputs are considered the effective bandwidth compression will be reduced as the $\mathrm{N}$ outputs correspond to a symbol duration of $\mathrm{T}$ and any increases in the number of samples beyond that is effectively an increase in the symbol duration and hence a larger $\alpha$.

The transmitter in Fig. 3 is effectively similar to the zero padding in IDFT evaluation when a higher frequency resolution is needed.

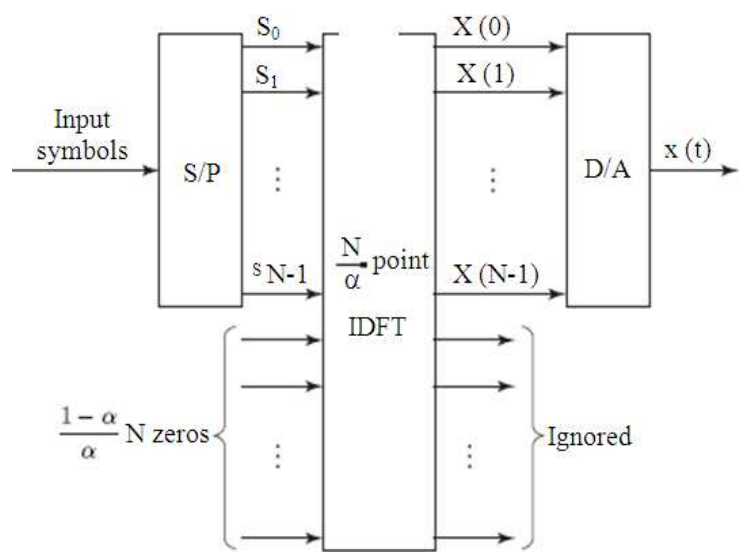

Fig. 3: The IDFT implementation for SEFDM proportional transmitter. Zeros are inserted after the input symbols to suppress unwanted frequency 


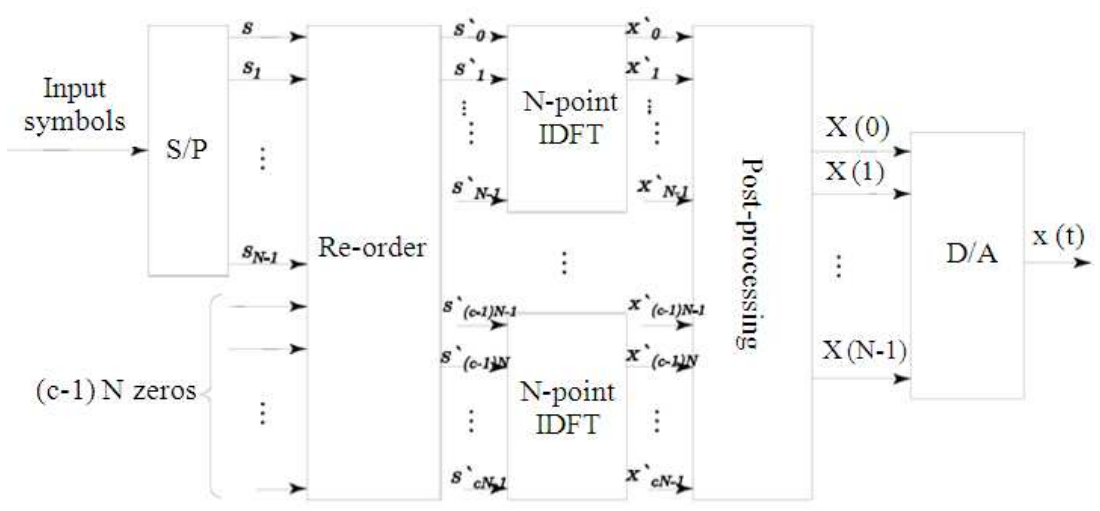

Fig. 4: Multiple IDFTs reduced complexity implementation for SEFDM transmitter

The difference is that only part of the generated IDFT is considered in order to maintain the characteristic relationship for SEFDM signals; $\Delta \mathrm{f}=\alpha / \mathrm{T}$. An advantage of this design is that the zeros could be positioned before, after or around the input symbols so as to decide the location of the SEFDM signal in the spectrum.

SEFDM as a sum of multiple IDFT: Manipulation of the SEFDM signal representation in (9) can lead to another new design that uses multiple shorter IDFT operations. In (9) it is shown that Eq. 10:

$$
\mathrm{X}[\mathrm{k}]=1 / \sqrt{\mathrm{N}} \sum_{\mathrm{m}=0}^{\mathrm{M}-1} \mathrm{~S}_{\mathrm{m}}^{\prime} \exp (2 \pi \mathrm{jmk} / \mathrm{M})
$$

Let $\alpha$ be a rational number, that is $\alpha=b / c$ for $b, c$ $2 \in \mathrm{N}$. Define the M long vector S' where Eq. 11:

$$
S^{\prime}(i)=\left\{\begin{array}{cc}
S_{i / b} & i \in I \\
0 & i \in Z \cap I
\end{array}\right.
$$

for $\mathrm{M}=\mathrm{cN}, \mathrm{Z}=[0,1, \cdots, \mathrm{M}-1]$ and $\mathrm{I}=[0, \mathrm{~b}, \cdots, \mathrm{b}$ $(\mathrm{N}-1)] . \mathrm{k}=0,1, \cdots, \mathrm{N}-1$. For such system settings $\mathrm{X}[\mathrm{k}]$ from (10) can be rearranged to Eq. 12:

$$
X[k]=\frac{1}{\sqrt{N}} \sum_{l=0}^{N-1} \sum_{i=0}^{c-1} s_{i+l c} \exp \left(\frac{j 2 \pi(i+l c) k}{c N}\right)
$$

by substituting by $\mathrm{m}=\mathrm{i}+\mathrm{lc}$. (12) can be further simplified to Eq. 13:

$$
X[k]=\frac{1}{\sqrt{N}} \sum_{i=0}^{c-1} \exp \left(\frac{j 2 \pi i k}{c N}\right) \sum_{l=0}^{N-1} s_{i+l c} \exp \left(\frac{j 2 \pi l K}{N}\right)
$$

The last term in (12) is the $\mathrm{N}$ long IDFT of the sequence $\mathrm{s}_{(\mathrm{i}+\mathrm{l}) \text {. }}$. This could be implemented by arranging the input as a $\mathrm{c} \times \mathrm{N}$ matrix in column major order, performing N-point IDFT on each row and multiplying the result by certain exponentials. In matrix format (12) can be represented as in (14), where $<[\cdot]$ denotes the diagonal elements of the argument matrix, ro $=\exp$ $\left(\frac{\mathrm{j} 2 \pi}{\mathrm{N}}\right)$ and $\nabla=\exp \left(\frac{\mathrm{j} 2 \pi}{\mathrm{cN}}\right)$.

Figure 4 shows how multiple shorter IDFTs are used in place of one large IDFT in Fig. 3. In the post processingblock the $\mathrm{k}^{\text {th }}$ outputs of the $\mathrm{c}$ IDFT stages are multiplied each by a complex exponential and combined to produceX $[\mathrm{k}]$. For each value there is a need to perform $\mathrm{c} N$-point IDFT operations and multiply by complex exponentials. The overall complexity of the system is expected to decrease as it uses multiple parallel shorter IDFT compared to the design in (9) and is demonstrated numerically. In addition, the complexity can be further reduced by generating the complex exponentials $\exp \left(\frac{\mathrm{j} 2 \pi \mathrm{ik}}{\mathrm{N}}\right)$ for $\mathrm{i}=$ $0, \cdots, \mathrm{c}-1$ and $\mathrm{k}=0, \cdots, \mathrm{N}$ beforehand by applying an IDFT (or more efficiently an IFFT) operation of length $\mathrm{cN}$ points initialized with $\{1,0,0, \cdots, 0\}$ for $\mathrm{c}$ circular shifts. The scheme could be especially useful for systems with large numbers of carriers as it becomes less feasible to use very long IDFT to generate the signal. Equation 12 shows that SEFDM could be effectively seen as a combination of multiple OFDM systems translated in frequency so as to reduce the spacing of the sub-carriers.

SEFDM DFT receiver: The SEFDM receiver consists of two main parts: the first extracts the statistics of the incoming signal and the second analyzes these statistics to produce an estimate of the transmitted signal. Originally the sufficient statistics are generated by correlating the incoming signal with orthonormal bases. 
The orthonormal bases can be obtained through the orthonormalization of the non-orthogonal carriers using Gram Schmidt technique and its modified versions. After that ML criterion is applied onto the sufficient statistics to estimate the originally sent symbols (Rodrigues and Darwazeh, 2003). In this study, we propose that the SEFDM signal is alternatively correlated with the conjugates of the original carriers instead of the orthonormal bases. The main advantage of this proposal is the elimination of the orthonormalization process which adds up to the complexity of the system.

Assuming that the received signal y $(\mathrm{t})$ is sampled to give the vector $\mathrm{Y}$ of size $\mathrm{N} \times 1$. Correlating this signal with the conjugate carriers will produce the statistics vector R such that Eq. 14-16:

$\left[\begin{array}{c}\mathrm{X}_{0} \\ \vdots \\ \mathrm{X}_{\mathrm{N}-1}\end{array}\right]=\angle\left[\begin{array}{cccc}{\left[\begin{array}{cccc}1 & 1 & \cdots & 1 \\ 1 & \text { ro } & \cdots & \mathrm{ro}^{\mathrm{N}-1} \\ \vdots & & \ddots & \\ 1 & \mathrm{ro}^{\mathrm{N}-1} & & \mathrm{ro}^{(\mathrm{N}-1) 2}\end{array}\right]} \\ {\left[\begin{array}{ccccc}\mathrm{s}_{0}^{\prime} & & \mathrm{s}_{1}^{\prime} & \cdots & \mathrm{s}_{\mathrm{c}-1}^{\prime} \\ \mathrm{s}_{\mathrm{c}} & \mathrm{s}_{\mathrm{c}+1}^{\prime} & & \mathrm{s}_{2 \mathrm{c}-1}^{\prime} \\ \vdots & & \ddots & \\ \mathrm{s}_{\mathrm{cN}-1 \mathrm{c}}^{\prime} & \cdots & & \mathrm{s}_{\mathrm{cN}-1}^{\prime}\end{array}\right]} \\ {\left[\begin{array}{ccccc}1 & 1 & \cdots & & 1 \\ 1 & \nabla & \cdots & \nabla^{\mathrm{N}-1} \\ \vdots & & \ddots & \vdots \\ 1 & \cdots & \cdots & \nabla^{(\mathrm{N}-1) 2}\end{array}\right]}\end{array}\right]$

$\mathrm{R}=\Phi * \mathrm{Y}$

$=\Phi^{*}(\Phi S+\mathrm{W})$

Based on the obtained statistics R, the detection of the sent symbols can be pursued. One possible solution is to search for the solution that satisfies Eq. 17:

$\operatorname{argmin}\|\mathrm{R}-\mathrm{CS}\|$

For S satisfying the constellation cardinality and $\mathrm{C}=\Phi^{*} \Phi$.

The DFT correlators: Applying the same concepts as, the correlation with the conjugate carriers can be realized with the Discrete Fourier Transform (DFT). The DFT of a sequence $\mathrm{Y}=\left[\mathrm{y}_{0}, \mathrm{y}_{1}, \cdots, \mathrm{y}_{\mathrm{N}-1}\right]$ ' denoted with the vector $\mathrm{F}\{\mathrm{Y}\}$ is given by (Oppenheim et al., 1999):

$F_{k}\{Y\}=\frac{1}{\sqrt{N}} \sum_{n=0}^{N-1} y_{n} \exp \left(\frac{-j 2 \pi n k}{N}\right)$

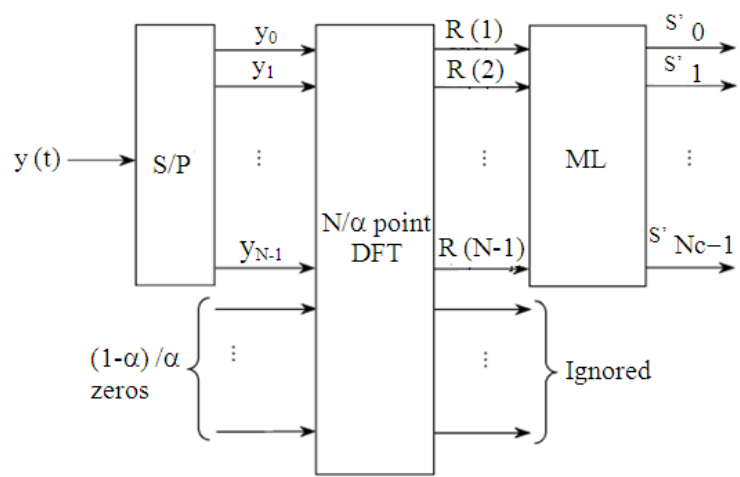

Fig. 5: DFT based SEFDM receiver

Hence (19) can be realized as Eq. 18:

$\mathrm{R}=(1 / \alpha) \mathrm{Q}^{*} \mathrm{Y}^{\prime}$

where, $\mathrm{Q}^{*}$ represents the $\mathrm{M}$ point DFT matrix for $\mathrm{M}=$ N/ $\alpha$ and Eq. 20:

$$
Y^{\prime}=\left\{\begin{array}{cc}
Y_{i} & 0 \leq \mathrm{i}<\mathrm{N} \\
0 & \mathrm{~N} \leq \mathrm{i}<\mathrm{M}
\end{array}\right.
$$

Or equivalently Eq. 21 and 22:

$R[k]=\frac{1}{\sqrt{N}} \sum_{i=0}^{c-1} \exp \left(\frac{j 2 \pi i k}{c N}\right) \sum_{l=0}^{N-1} Y^{\prime}(i+l c) \exp \left(\frac{j 2 \pi l k}{N}\right)$

Where:

$$
Y^{\prime}(i)=\left\{\begin{array}{cc}
Y_{i / b} & i \in I \\
0 & i \in Z \cap I
\end{array}\right.
$$

Figure 5 and 6, Depict receivers structures based on (19) and (21). The two designs show how the correlation with the conjugate carriers is realized with standard DFT blocks. The generated statistics are fed to an ML detector to estimate the transmitted symbols.

Performance analysis: Section presented three SEFDM transmitters based on the IDFT. The spectrum of the digitally generated signal by any of the three transmitters is presented in Fig. 7. It is clear that the transmitters provided the required spectrum compression as the signal generated by a bank of modulators. When $\alpha=1$, the proposed transmitters will automatically reduce to the well known OFDM IDFT based system. It is worth noting that for any arbitrary sampling rate the transmitters described here will produce the required compressed spectrum of SEFDM system. 
Am. J. Engg. \& Applied Sci., 4 (4): 598-606, 2011

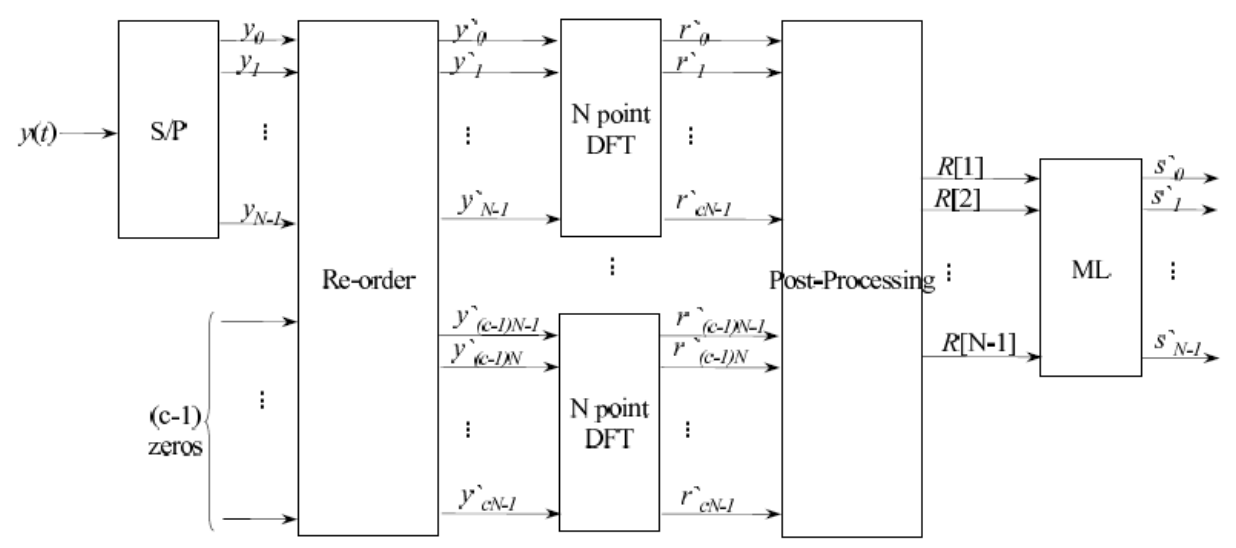

Fig. 6: Multiple IDFTs reduced complexity implementation for SEFDM receiver

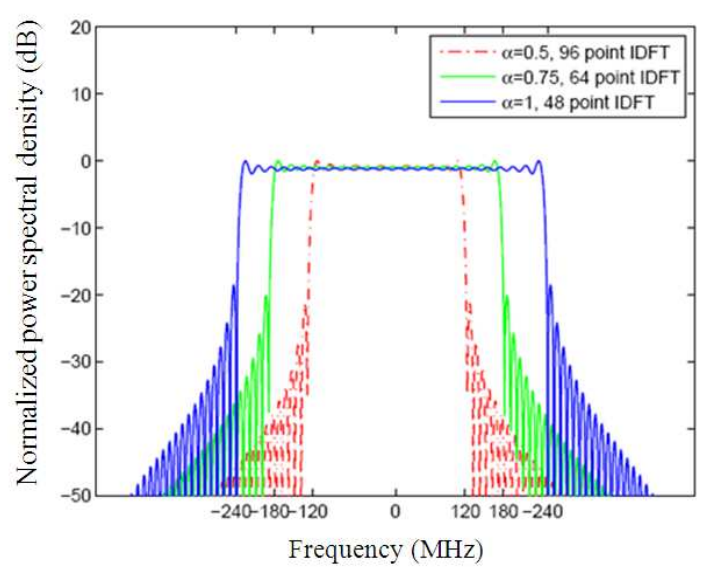

Fig. 7: IDFT SEFDM spectrum for 48 carriers for $\alpha=$ $0.5,0.75$ and 1

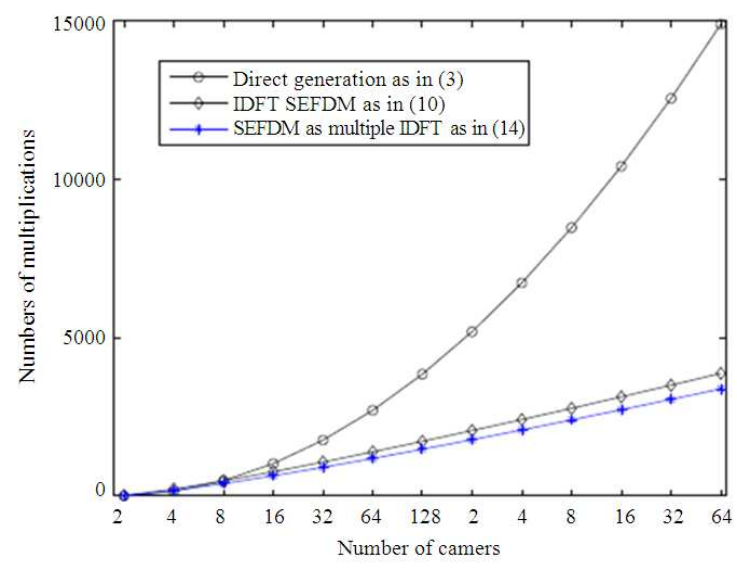

Fig. 8: Computational complexity for the transmitters in section, for $\alpha=2 / 3$ and $\gamma=2$
The main differences between the proposed designs section are in the size and number of IDFT modules in the transmitter. The design in (9) uses a single IDFT module of size $\mathrm{N} / \alpha$, hence, requires that $\mathrm{N} / \alpha$ is an integer number. The design in section uses multiple IDFT modules which are shorter in length.

Differences in the number and size of the IDFT in the proposed design will offer different computational complexity. The computational complexity of the directly generated SEFDM signal as in (2) is $\mathrm{N}^{2}$ complex multiplications and $\mathrm{N}$ (N-1) complex additions. The complexity of the proposed transmitters in section is expected to be much lower than the direct generation of the signal as the complexity of the designs is of $\mathrm{O}(\mathrm{N} \log \mathrm{N})$ when the IDFT is economically evaluated by Fast Fourier Transform (FFT) algorithm. Assuming that the complexity of FFT algorithm is $\gamma \mathrm{N} \log _{2} \mathrm{~N}$ complex multiplications and additions, where $\gamma$ is a constant; the complexity of the transmitter in section will be Eq. 23:

$\gamma\left(\mathrm{N} / \alpha \log _{2} \mathrm{~N} / \alpha\right)=\gamma\left(\mathrm{N} / \alpha \log _{2} \mathrm{~N}-\mathrm{N} / \alpha \log _{2} \alpha\right)$

Section described a reduced complexity design for $\alpha=b / c$. The computational complexity of this design is a combination of the complexity of the $\mathrm{cN}$ long IDFT plus $\mathrm{N}$ complex multiplications and (c-1) $\mathrm{N}$ complex additions. Figure 8 shows the complexity of the proposed systems in sections by plotting the number of multiplicationswith respect to the number of carriers for a given $\alpha$ as an example of the expected computational savings for the proposed systems. It is clear that the proposed systems provide substantial computational savings especially for large system sizes when compared with the direct generation of the signal. 
Am. J. Engg. \& Applied Sci., 4 (4): 598-606, 2011

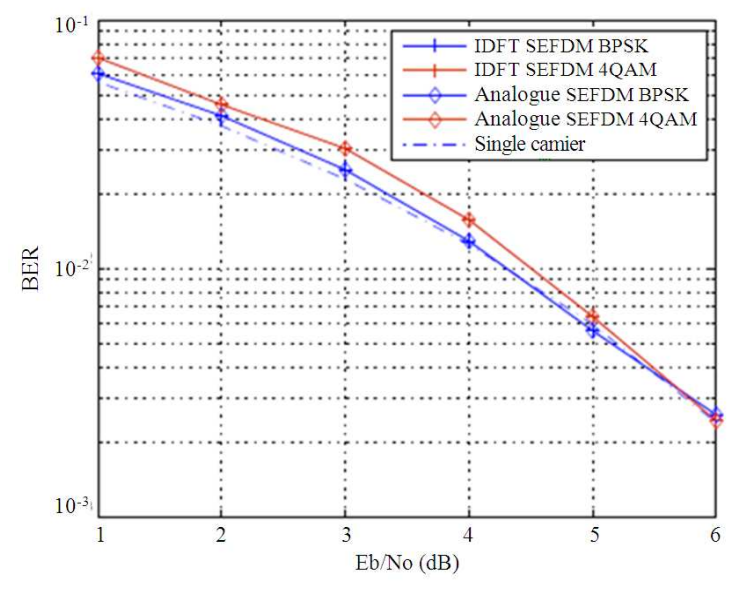

Fig. 9: BER performance of IDFT based SEFDM system for 8 carrier system, $\alpha=0.8$ and BPSK and 4QAM modulating symbols

The time domain signal is expected to slightly differ from the SEFDM signal generated in (1) depending on the size of the IDFT used, as an expected consequence of different sampling resolution in time domain. Nevertheless, the generated signal would have the required compressed spectrum as depicted in Fig. 7. The outputs of the transmitter, which are time samples, may be chosen in a way that eases their demodulation and allow for post coding of these samples. Reception of the signals generated could follow any method used to receive the original signal described in (Kanaras et al., 2008b).

Finally a complete SEFDM system is simulated in AWGN channel as in (Rodrigues and Darwazeh, 2003). Detection is realized by projecting the signal onto the conjugate carriers realized with the DFT blocks as described in section followed by ML detector.

Figure 9 shows numerical results for the BER of the digitally generated and received SEFDM signal. The digital signal exhibited the same BER as the analogue one. From BER results it is confirmed that the new way of generating and receiving the SEFDM signal exhibited the same BER performance as the analogue way.

\section{CONCLUSION}

In this study a new framework to generate Spectrally Efficient System signals is proposed. The framework is based on using standard IDFT to generate fractionally spaced signals in frequency. The proposed framework eliminates the need for a bank of analogue modulators, allowing for an easy generation of SEFDM signals for a flexible range of frequency separation. The equivalence of the digitally generated signal and the oscillator based one is proved mathematically. Numerical verifications confirmed that the transmitters produce the required bandwidth compression with the same.

BER performance as the oscillator based signal. The structure of the proposed transmitters employ similar building blocks as OFDM systems, hence, will facilitate a smooth changeover between existing systems based on OFDM to systems employing SEFDM. Moreover, systems that allow for multi-rate communications could benefit from these methods where the operation could be switched between OFDM and SEFDM. Such feature could be especially useful for applications where the available spectrum changes. Such mode of operation can be more suited for the uplink as the base station is more capable to accommodate the complex SEFDM receiver. The main limitation of these simple transmitters is that it may require a larger IDFT when compared with OFDM. Nevertheless, when compared with generation with bank of modulators, these methods will result in reduced cost of the system. In addition, the proposed framework is extended to the reception side leading to the proposal of the DFT to extract the sufficient statistics of the incoming signal. Numerical results has confirmed similar BER performance to the correlators based signal. Further enhancement may be sought by exploring mechanisms to simplify the IDFT/DFT design of SEFDM system in order to present SEFDM system as a realistic competitor for next generation communications.

\section{ACKNOWLEDGMENT}

Safa Ahmed is grateful to UCL for funding her $\mathrm{PhD}$ studies through Overseas Research Student Award (ORS) and UCL Graduate School Research Scholarship (GSRS).

\section{REFERENCES}

Bailey, D.H. and P.N. Swarztrauber, 1991. The fractional fourier transform and applications. SIAM Rev., 33: 389-404.

Chorti, A., I. Kanaras, M.R.D. Rodrigues and I. Darwazeh, 2010. Joint channel equalization and detection of spectrally efficient FDM signals. Proceedings of the IEEE 21st International Symposium on Personal Indoor and Mobile Radio Communications, Sept. 26-30, IEEE Xplore Press, Instanbul, pp: 177-182. DOI: 10.1109/PIMRC.2010.5671644 
Hamamura, M. and S. Tachikawa, 2004. Bandwidth efficiency improvement for multi-carrier systems. Proceeding of the 15th IEEE International Symposium on Personal, Indoor and Mobile Radio Communications, Sept. 5-8, IEEE Xplore Press, pp: 48-52. DOI: 10.1109/PIMRC.2004.1370834

Hanzo, L. and T. Keller, 2006. OFDM and MC-CDMA: A Primer. 1st Edn., John Wiley, Piscataway, New Jersey, ISBN-10: 0470030070, pp: 411.

Hanzo, L., 2003. OFDM and MC-CDMA for Broadband Multi-user Communications. 1st Edn., J. Wiley, Chichester, ISBN-10: 0470858796, pp: 978.

Kanaras, I., A. Chorti, M. Rodrigues and I. Darwazeh, 2008a. Analysis of Sub-optimum detection techniques for a bandwidth efficient multi-carrier communication system. Proceedings of the Cranfield Multi-Strand Conference, May 6-7, Cranfield University.

Kanaras, I., A. Chorti, M. Rodrigues and I. Darwazeh, 2008b. An optimum detection for a spectrally efficient non orthogonal FDM system. Proceedings of the 13th International OFDM Workshop, (OFDMW' 08), Hamburg.

Kanaras, I., A. Chorti, M. Rodrigues and I. Darwazeh, 2008c. A combined MMSE-ML detection for a spectrally efficient non orthogonal FDM signal. Proceedings of the 5th International Conference on Broadband Communications, Networks and Systems, Sept. 8-11, IEEE Xplore Press, London, pp: 421-425. DOI: 10.1109/BROADNETS.2008.4769119

Kanaras, I., A. Chorti, M.R.D. Rodrigues and I. Darwazeh, 2009. Spectrally efficient FDM signals: Bandwidth gain at the expense of receiver complexity. Proceedings of the IEEE International Conference on Communications, Jun. 14-18, IEEE Xplore Press, Dresden, pp: 1-6. DOI: 10.1109/ICC.2009.5199477

Macedo, A.S. and E.S. Sousa, 1997. Coded OFDM for broadband indoor wireless systems. Proccedings of the IEEE International Conference on Towards the Knowledge Millennium Communications, Jun. 812, IEEE Xplore Press, Montreal, Que., pp: 934938. DOI: 10.1109/ICC.1997.610016
Mazo, J.E., 1975. Faster than Nyquist Signalling. Bell Syst. Technol. J., 54: 429-458.

Oppenheim, A.V., R.W. Schafer and J.R. Buck, 1999. Discrete-Time Signal Processing. 2nd Edn., Prentice Hall, New Jeresy, ISBN-10: 0137549202, pp: 870.

Proakis, J.G. and M. Salehi, 2008. Digital Communications. 5th Edn., McGraw-Hill, Boston, ISBN-10: 0071263780, pp: 1150

Raleigh, G.G. and J.M. Cioffi, 1998. Spatio-temporal coding for wireless communication. IEEE Trans. Commun., 46: 357-366.

Rodrigues, M.R.D. and I. Darwazeh, 2002. Fast OFDM: A proposal for doubling the data rate of OFDM schemes. Proceedings of the International Conference on Telecommunications, (ICT' 02), Beijing, China, pp: 484-487.

Rodrigues, M.R.D. and I. Darwazeh, 2003. A spectrally efficient frequency division multiplexing based communication system. Proceedings of the 8th International OFDM Workshop, (IOW' 03), Hamburg, Germany, pp: 70-74.

Rusek, F. and J.B. Anderson, 2005. The two dimensional Mazo limit. Proceedings of the International Symposium on Information Theory, Sept. 4-9, IEEE Xplore Press, Adelaide, SA., pp: 970-974. DOI: 10.1109/ISIT.2005.1523482

Rusek, F. and J.B. Anderson, 2009. Multistream faster than nyquist signaling. IEEE Trans. Commun., 57: 1329-1340. 10.1109/TCOMM.2009.05.070224

Salz, J. and S.B. Weinstein, 1969. Fourier transform communication system. Proceedings of the 1 st ACM Symposium on Problems in the Optimization of Data Communications Systems, (PODCS' 69), ACM Press, New York, pp: 99-128. DOI: 10.1145/800165.805240

Weinstein, S. and P. Ebert, 1971. Data transmission by frequency-division multiplexing using the discrete fourier transform. IEEE Trans. Commun. Technol., 19: 628-634. DOI: 10.1109/TCOM.1971.1090705

Xiong, F., 2003. M-ary amplitude shift keying OFDM system. IEEE Trans. Commun., 51: 1638-1642. DOI: 10.1109/TCOMM.2003.818103 